\title{
Supersoft Top Squarks
}

\author{
Timothy Cohen $\odot,{ }^{1}$ Nathaniel Craig, ${ }^{2}$ Seth Koren $\odot,{ }^{2}$ Matthew McCullough, ${ }^{3,4}$ and Joseph Tooby-Smith $\odot^{5}$ \\ ${ }^{1}$ Institute for Fundamental Science, Department of Physics, University of Oregon, Eugene, Oregon 97403, USA \\ ${ }^{2}$ Department of Physics, University of California, Santa Barbara, California 93106, USA \\ ${ }^{3}$ Theoretical Physics Department, CERN, CH-1211 Geneva 23, Switzerland \\ ${ }^{4}$ DAMTP, University of Cambridge, Wilberforce Road, Cambridge CB3 OWA, United Kingdom \\ ${ }^{5}$ Cavendish Laboratory, University of Cambridge, J. J. Thomson Avenue, Cambridge CB3 OHE, United Kingdom
}

(Received 26 May 2020; accepted 26 August 2020; published 7 October 2020)

\begin{abstract}
In a supersymmetric theory, the IR contributions to the Higgs mass are calculable below the mediation scale $\Lambda_{\mathrm{UV}}$ in terms of the IR field content and parameters. However, logarithmic sensitivity to physics at $\Lambda_{\mathrm{UV}}$ remains. In this Letter, we present a first example of a framework, dictated by symmetries, to supersoften these logarithms from the matter sector. The result is a model with finite, IR-calculable corrections to the Higgs mass. This requires the introduction of new fields- the "lumberjacks"-whose role is to screen the UV-sensitive logs. These models have considerably reduced fine-tuning, by more than an order of magnitude for high-scale supersymmetry. This impacts interpretations of the natural parameter space, suggesting it may be premature to declare a naturalness crisis for high-scale supersymmetry.
\end{abstract}

DOI: $10.1103 /$ PhysRevLett.125.151801

The search for models of short distance physics that yield a calculable Higgs mass continues to be one of the most important motivations for explorations beyond the standard model. This reductionist approach to fundamental questions has many precedents. The scalar mass in the LandauGinzburg theory is calculable utilizing the more microscopic BCS theory of superconductivity. Hadron masses derive from the underlying QCD quark mass and gauge coupling parameters. Yet, despite our best efforts, no experimental hints towards the provenance of the Higgs mass have emerged, leading to the commonly held belief that the standard model must be fine-tuned. Here, via a novel extension of the standard model matter sector, we challenge the notion that the absence of evidence for naturalness (thus far) is evidence of its absence.

Taking seriously the notion that the Higgs mass parameter $M_{H}$ originates at some microscopic scale " $\Lambda_{\mathrm{UV}}$," along with the observed lack of protective symmetries within the standard model, results in the expectation that $M_{H}$ should be parametrically determined by $\Lambda_{\mathrm{UV}}$. Scenarios that can accommodate a comfortable separation $M_{H} \ll \Lambda_{\mathrm{UV}}$ rely on invoking additional symmetries, which imply the existence of associated fields that are required to fill out complete representations. Since the top quark has the largest coupling to the Higgs boson, one expects that the

Published by the American Physical Society under the terms of the Creative Commons Attribution 4.0 International license. Further distribution of this work must maintain attribution to the author(s) and the published article's title, journal citation, and DOI. Funded by SCOAP . relationship between $M_{H}$ and $\Lambda_{\mathrm{UV}}$ is dominated by new physics in the top sector. The best studied examples are supersymmetry (SUSY), which introduces the colored scalar top squarks, or spontaneously broken global symmetries, which requires a set of colored fermionic top partner fields. If the mass scale of such fields is $\tilde{m}$ then the underlying dependence of the Higgs mass on the UV physics at $\Lambda_{\mathrm{UV}}$ is softened from a quadratic sensitivity $\delta M_{H}^{2} \propto \Lambda_{\mathrm{UV}}^{2}$, to a logarithmic one $\delta M_{H}^{2} \propto \tilde{m}^{2} /$ $\left(16 \pi^{2}\right) \log \left(\Lambda_{\mathrm{UV}} / \tilde{m}\right)$. This remaining logarithmic sensitivity to $\Lambda_{\mathrm{UV}}$ betrays the fact that although $\tilde{m} \ll \Lambda_{\mathrm{UV}}$ can be naturally accommodated, the Higgs mass is only truly calculable if one knows the complete theoretical picture at $\Lambda_{\mathrm{UV}}$. The implications of this residual UV dependence for the naturalness of the weak scale can be profound, heightening the sensitivity of the Higgs mass to the scale of symmetry breaking.

In this Letter, we investigate to what extent the scaling $\delta M_{H}^{2} \propto \tilde{m}^{2} /\left(16 \pi^{2}\right) \log \left(\Lambda_{\mathrm{UV}} / \tilde{m}\right)$ is inevitable. Instead of demanding that the mass scale $\tilde{m}$ is small to preserve naturalness, we reorient the question by instead asking if it is possible that the logarithm can be rendered insensitive to the UV scale. Specifically, we will introduce scenarios in which an additional symmetry-enforced cancellation occurs over a large range of scales, essentially screening the Higgs mass from $\tilde{m}$-dependent corrections all the way from the microscopic scale $\Lambda_{\mathrm{UV}}$ (where the symmetry breaking is generated) down to the $\mathrm{TeV}$ scale. Corrections are still present at all scales, as expected for logarithmic UV sensitivity. However, a UV conspiracy enforces an additional cancellation to remove logs, a phenomenon we will call "logging." In practice, this amounts to creating an equal-and- 


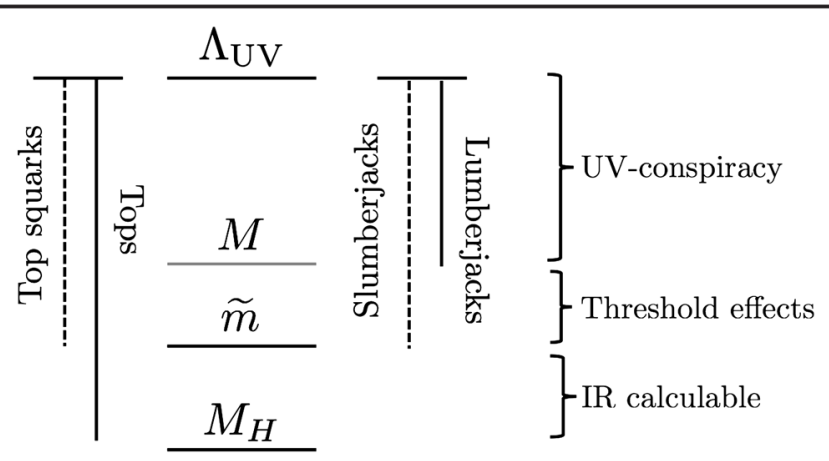

FIG. 1. A sketch of the scales relevant to the broad picture, with the running contributions depicted on either side of the mass scales. Above the scale $M$ the lumberjack fields conspire with the MSSM states to screen running Higgs mass corrections from the UV-scale $\Lambda_{\mathrm{UV}}$ all the way down to the IR. Calculable threshold corrections to the Higgs mass are generated below the mass scale $M$. Scalars (dashed) are separated from their partner fermions (solid) by an equal and opposite-signed soft-mass squared.

opposite "little anti-hierarchy problem," that largely annihilates the little hierarchy problem typically present in SUSY models. The framework is depicted in Fig. 1.

Importantly, logging away all UV sensitivity implies that the Higgs mass corrections must be fully calculable in terms of IR parameters. Some examples of IR determined contributions to the Higgs mass already exist in the literature. In perhaps the most familiar class of models, of which logging is a member, symmetries orchestrate screening among the IR degrees of freedom. For example, in "supersoft" theories with Dirac Gauginos, the one-loop gauge corrections to scalar masses are finite [1]—one way to view the model presented here is that it demonstrates how to supersoften the matter sector. In maximally symmetric pseudo-Nambu-Goldstone boson Higgs models [2], a remnant symmetry renders the Higgs potential finite (see also [3] for work which reduces logarithms in pseudoNambu-Goldstone boson models). A second class of models are those in which there are simply no local operators that could contribute to the Higgs mass at $\Lambda_{\mathrm{UV}}$, and hence no logarithms are associated with their evolution. In models of Scherk-Schwarz SUSY breaking, locality ensures that all scalar mass corrections are finite and calculable [4,5], albeit with coefficients that are relatively large with respect to the mass of the lightest colored state and an infinite number of extra states required for the mechanism to be operative. These finite corrections have for some time been employed with respect to the Higgs mass [6-11] and the absence of large logarithms is still attractive with respect to questions of naturalness in supersymmetric theories [12]. In little Higgs models $[13,14]$, collective symmetry breaking and the attendant nonlocality in theory space results in one-loop finite mass corrections. Alternatively, one can mimic IR domination by considering UV-sensitive models with a very low $\Lambda_{\mathrm{UV}}$, which by design implies small logarithms, yielding reduced fine-tuning for the viable parameter space.

A common aspect of many of these IR-dominated models is that symmetries and/or locality forbid the possibility of local counterterms which correct the Higgs mass, rendering the Higgs mass IR calculable. Motivated by this observation, our purpose here is to introduce a new class of supersymmetric effective field theories, in which the interplay of global exchange symmetries and symmetry breaking forbid these local counterterms. The result will be that incalculable logarithms are screened, such that weakscale fine-tuning is significantly reduced.

Logging SUSY.-In this section, we will present a low energy SUSY EFT with supersoft top squarks. We add a complete copy of the third generation matter, the "lumberjack" fields whose purpose is to remove the logs (one could duplicate all of the minimal supersymmetric standard model (MSSM) matter in the same way, with a minimal impact on the resulting tuning). These lumberjacks are related to the third generation matter by an exchange symmetry. As a result, the most general matter-sector renormalizable superpotential is

$$
W_{\lambda}=\lambda_{t} H_{u} Q U^{c}+\lambda_{t} H_{u} Q^{\prime} U^{\prime c} .
$$

To lift the lumberjack fields, they are paired with an additional set of vectorlike partners. A nonzero vectorlike mass $M$ softly breaks the exchange symmetry:

$$
\boldsymbol{W}_{\boldsymbol{M}}=M\left(\boldsymbol{Q}^{\prime} \overline{\boldsymbol{Q}}^{\prime}+\boldsymbol{U}^{\prime c} \overline{\boldsymbol{U}}^{\prime c}\right) .
$$

Note that the exchange symmetry is not broken by the spectrum itself, since $\overline{\boldsymbol{Q}}^{\prime}$ and $\overline{\boldsymbol{U}}^{\prime c}$ do not transform.

The matter squarks and their scalar lumberjack partners (the "slumberjacks") are given the following exchangebreaking soft masses:

$$
V_{\text {Soft }} \simeq \tilde{m}^{2}\left(|\tilde{Q}|^{2}+\left|\tilde{U}^{c}\right|^{2}-\left|\tilde{Q}^{\prime}\right|^{2}-\left|\tilde{U}^{\prime c}\right|^{2}\right),
$$

where tachyonic scalar masses are avoided due to the additional vectorlike mass contribution of Eq. (2). We will show how one can obtain this SUSY breaking pattern from the UV, which will make concrete the interpretation of $\tilde{m}^{2}$ as a spurion for the simultaneous spontaneous breaking of SUSY and the exchange symmetry [15].

We then compute the masses, from which we obtain the Higgs mass corrections from both the top-top squark and lumberjack sectors:

$$
\begin{aligned}
\delta M_{H_{u}}^{2}= & -\frac{3 \lambda_{t}^{2}}{8 \pi^{2}} \tilde{m}^{2}\left[R+(R-1)^{2} \log (R-1)\right. \\
& -(R-2) R \log (R)] .
\end{aligned}
$$

where $R=M^{2} / \tilde{m}^{2}$. Due to the presence of the lumberjacks, the UV sensitivity has been logged away, leaving 
behind only the IR contribution, as expected. To leading order in $1 / R$ we have

$$
\delta M_{H_{u}}^{2} \simeq-\frac{3 \lambda_{t}^{2}}{8 \pi^{2}} \tilde{m}^{2}\left[\frac{3}{2}+\log \left(\frac{M^{2}}{\tilde{m}^{2}}\right)\right],
$$

which makes clear that, up to an additional finite threshold correction, the scale $M$ effectively replaces the UV cutoff, which is consistent with the simple renormalization group evolution interpretation of Fig. 1.

More generally, one might imagine extending the third generation matter to include a variety of fields $\Phi_{i}$ with diverse standard model charges and couplings to the Higgs,

$$
\boldsymbol{W}=\frac{1}{2} \lambda_{H}^{i j} \boldsymbol{H}_{u} \Phi_{i} \Phi_{j}+\frac{1}{2} M^{i j} \Phi_{i} \Phi_{j},
$$

as well as soft terms

$$
V_{\mathrm{Soft}}=\frac{1}{2}\left(\tilde{m}^{2}\right)_{i}^{j} \tilde{\Phi}^{* i} \tilde{\Phi}_{j}
$$

The general condition for screening UV-sensitive logs at one loop is simply $\lambda_{H k i} \lambda_{H}^{k j}\left(\tilde{m}^{2}\right)_{j}^{i}=0$. When all fields couple with equal strength to the Higgs, this reduces to the simple condition that the trace of the soft masses vanishes, $\left(\tilde{m}^{2}\right)_{i}^{i}=0$; this is clearly satisfied by the soft terms in Eq. (3).

Fine-tuning: As in the MSSM, to realize the observed Higgs mass one would likely need additional contributions to the Higgs quartic, such as radiative corrections from $A$ terms or by extending the framework to include additional next-to-minimal supersymmetric standard model (NMSSM)-like singlets. As a result, any exploration of the parameter space realizing the observed Higgs mass is inherently model dependent. However, independent of these considerations, we may still study the improvement in fine-tuning relative to a standard MSSM-like scenario. Whenever the fine-tuning is dominated by the presence of a heavy top squark in the MSSM, the quadratic corrections to the up-type Higgs mass give the leading contribution:

$$
\delta M_{H_{u}}^{2}=-\frac{3 \lambda_{t}^{2}}{8 \pi^{2}} \tilde{m}^{2} \log \left(\frac{\Lambda_{\mathrm{UV}}^{2}}{\tilde{m}^{2}}\right),
$$

which must be tuned against other contributions, such as the $\mu$ term. On the other hand, the corrections in the logged model are given in Eq. (4). As a result, regardless of how the required quartic is generated, if we simply assume that the fine-tuning is dominated by the top squark sector, we can estimate the gain in fine-tuning by computing the ratio of these two corrections as a function of the mass of the lightest colored scalar.

In Fig. 2, we show contours of the improvement in the tuning when the lumberjacks are present as compared to the MSSM in the $R$ versus $\tilde{m}$ plane. Even for low mediation scales, it is clear that the reduction in the tuning is

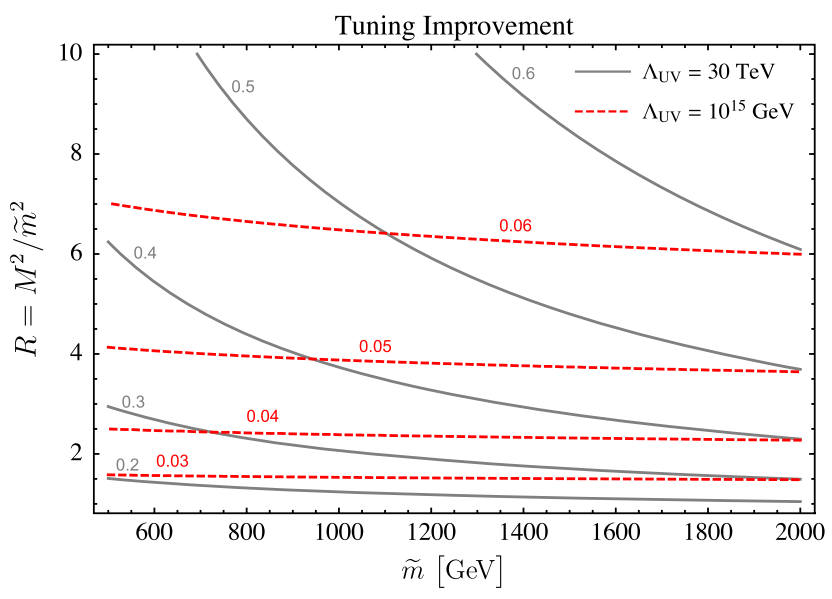

FIG. 2. Contours of the Higgs mass squared corrections in the logged model [Eq. (4)] divided by the corrections in the MSSM [Eq. (8)], in the $R=M^{2} / \tilde{m}^{2}$ versus $\tilde{m}$ plane. The gray contours are for $\Lambda_{\mathrm{UV}}=30 \mathrm{TeV}$ and red for $\Lambda_{\mathrm{UV}}=10^{15} \mathrm{GeV}$, which is relevant for the MSSM corrections. Thus these contours represent the reduction in fine-tuning in the supersoft model relative to the MSSM. We find improvement factors as large as 30 for high mediation scales.

considerable. For instance, if one has a grand unified theory-scale model compatible with current bounds on top squarks with tuning of $\mathcal{O}(1 \%)$, then the supersoft version could be realized with a rather negligible tuning of $\mathcal{O}(30 \%)$ for low $R$. Indeed, fine-tuning in logged models approaches the favorable level obtained if one used only the infrared values of supersymmetry-breaking parameters [18].

Although simplistic, the analysis captures the leading fine-tuning aspects, though effects beyond leading logarithm are likely to moderate the improvement $[19,20]$. Looking forward, it would be interesting to revisit the current fine-tuning in supersymmetric models with high scale mediation for a logged model to quantitatively assess the level of pressure on supersymmetric naturalness.

It bears noting that the radiative correction to the Higgs quartic from the lumberjack fields is negative and proportional to $\log M / \tilde{m}$. This reduces the overall contribution from the top sector in close analogy with the reduction of the $D$-term quartic in supersoft theories with Dirac gauginos. A more rigorous treatment would reveal a trade-off between the reduction in the log contribution (approximately $\left.\log \Lambda_{\mathrm{UV}} / M\right)$ and the decreased quartic when the observed Higgs mass is driven by the top sector. In this case, the naturalness improvement will be significant as long as $\tilde{m} \Lambda_{\mathrm{UV}} \gg M^{2}$.

UV completions. - We assume that the SUSY breaking soft masses arise from a superfield $D$ term $\langle\boldsymbol{\Phi}\rangle=D_{\Phi} \theta^{2} \bar{\theta}^{2}$, which is odd under the exchange symmetry, $\Phi \rightarrow-\boldsymbol{\Phi}$. The Kähler potential at the matching scale $\Lambda_{\mathrm{UV}}$ is

$$
\boldsymbol{K}=\frac{\boldsymbol{\Phi}}{\Lambda_{\mathrm{UV}}^{2}}\left(|\boldsymbol{Q}|^{2}+\left|\boldsymbol{U}^{c}\right|^{2}-\left|\boldsymbol{Q}^{\prime}\right|^{2}-\left|\boldsymbol{U}^{\prime c}\right|^{2}\right) .
$$


Finiteness of the Higgs mass corrections follows from the fact that the Higgs cannot couple to the SUSY breaking field in the Kähler potential as $K \supset \boldsymbol{\Phi}|\boldsymbol{H}|^{2}+$ H.c., since the global $\mathcal{Z}_{2}$ symmetry forbids any counterterm at this order.

While the EFT is self-consistent, it is interesting to consider the possible UV structure. One possibility would be to realize $\boldsymbol{\Phi}$ as a pair of partner fields which swap under the exchange symmetry

$$
\boldsymbol{\Phi}=|\boldsymbol{X}|^{2}-|\overline{\boldsymbol{X}}|^{2} .
$$

The sign enforces consistency with the coupling in Eq. (9). An $F$ term of this form could be generated in a number of ways. One would be to have $\boldsymbol{W}_{\boldsymbol{X}}=f \boldsymbol{X}$. Another viable approach would be to utilize an O'Raifeartaigh-like superpotential [21] with a small source of exchange symmetry breaking to yield the desired $F$ term.

It is also important to understand the impact of additional operators that could be present in the UV. Perhaps the most concerning coupling is

$$
\boldsymbol{K}_{\boldsymbol{H}}=\frac{|\boldsymbol{X}|^{2}+|\overline{\boldsymbol{X}}|^{2}}{\Lambda_{H}^{2}}|\boldsymbol{H}|^{2},
$$

which is allowed by the symmetries and would spoil the supersoftness. Therefore, our IR analysis is valid under the assumption that the leading coupling is to the matter fields, as in Eq. (9). Then the generation of the dangerous coupling in Eq. (11) would be suppressed by extra powers of $\tilde{m}^{2} / \Lambda_{\mathrm{UV}}^{2}$, or by some number of loop factors. Both of these contributions are consequently highly UV dependent.

An alternative UV scenario introduces an additional anomalous $\mathrm{U}(1)_{X}$ gauge symmetry under which the MSSM and lumberjack superfields carry equal and opposite charges. These assignments are consistent with the exchange symmetry if the vector superfield is odd $\boldsymbol{V} \rightarrow-\boldsymbol{V}$. The $\mathrm{U}(1)_{X}$ can be spontaneously broken supersymmetrically if a pair of superfields $\boldsymbol{X}$ and $\overline{\boldsymbol{X}}$ with equal and opposite charge have vevs. Integrating out the vector multiplet could be responsible for generating Eq. (9). Since these fields also transform under the exchange symmetry, they may be used to generate the exchange-symmetryrespecting superpotential Yukawa couplings given in Eq. (1). The exchange-breaking superpotential masses in Eq. (2) may be generated by a small exchange-breaking Yukawa coupling involving $\boldsymbol{X}$ and the lumberjacks. Finally, the construction of [22], wherein an anomalous $\mathrm{U}(1)_{X}$ symmetry obtains a supersymmetry-breaking $D$ term well below the scale of spontaneous gauge symmetry breaking, could provide the source of the soft masses in Eq. (3).

Gauginos: In the lumberjacking and supersoft spirit we opt to have Dirac gauginos, since this generates one-loop finite radiative corrections in the scalar sector [1] (Majorana gaugino masses would spoil the scalar supersoftness at oneloop, with large group theory factors, reintroducing logarithms and spoiling the improvements in naturalness for any significant separation between the SUSY-breaking and EW scales). This can be realized consistently with the existing setup since the adjoint chiral superfields $\boldsymbol{A}_{j}$ may be made odd under the exchange symmetry, such that a Dirac gaugino mass may be generated through the usual operator

$$
\boldsymbol{W}_{\text {Gauge }}=\frac{1}{\Lambda_{\mathrm{UV}}} \boldsymbol{W}_{\alpha}^{\boldsymbol{\Phi}} \boldsymbol{W}_{j}^{\alpha} \boldsymbol{A}_{j},
$$

where $\boldsymbol{W}_{\alpha}^{\Phi}$ is the vector chiral superfield for $\Phi$ whose $D$ term vev yields the Dirac mass, and $j$ denotes the choice of gauge group. The $\mathcal{Z}_{2}$ symmetry acts on the adjoint chiral superfield as $\boldsymbol{A}_{j} \rightarrow-\boldsymbol{A}_{j}$. Since the IR exchange symmetry is respected by the gauge sector, the arguments for the scalar sector given in the previous section are unmodified. The dangerous supersoft-spoiling mass terms associated with the $\mu-B_{\mu}$ problem of Dirac gaugino scenarios may be avoided through the GoGa mechanism [23,24].

Higgs quartic: To raise the Higgs quartic without introducing UV-sensitive logarithms we may also supersoften the singlet sector of the NMSSM. To do this we follow the spirit of the top sector and introduce two singlets with an exchange symmetry

$$
\boldsymbol{W}_{S}=\lambda\left(\boldsymbol{S}+\boldsymbol{S}^{\prime}\right) \boldsymbol{H}_{u} \boldsymbol{H}_{\boldsymbol{d}}+\frac{1}{2} M_{S}\left(\boldsymbol{S}^{2}+\boldsymbol{S}^{\prime 2}\right) .
$$

If desired, additional cubic interactions can be added consistent with the exchange symmetry. To introduce SUSY breaking we include an exchange-breaking soft term,

$$
V_{\text {Soft }} \simeq-\tilde{m}_{S}^{2}\left(|\tilde{S}|^{2}-\left|\tilde{S}^{\prime}\right|^{2}\right),
$$

with a similar origin to the soft masses in the top squark sector. As before, the one-loop radiative corrections will be IR calculable, allowing to raise the Higgs mass without the concern of large logarithms. Furthermore, since in the supersoft model the quadratic corrections tend to be smaller than standard scenarios with even a very low messenger scale, it is likely that the fine-tuning outcome for the singlet sector will be less severe than in standard NMSSM scenerios [25].

Phenomenology.-Much of the LHC phenomenology is driven by the Dirac nature of the gauginos. Studies of the novel signatures of Dirac gauginos have been undertaken previously [26-29]. Due to the supersoftness, the gluinos can be heavier than in the MSSM without being the dominant source of tuning, which implies that jets plus missing energy signatures from the gluino and light squark sector have a lower rate, see, e.g., [30].

The novel feature of this model is the presence of the vector-like lumberjacks. Note that in the model as described in the first section, these fields would be stable and hence, depending on the cosmological history, 
potentially phenomenologically unacceptable. To complete the story, one could introduce small $\mathcal{Z}_{2}$ breaking terms that enable lumberjack decays. Since this coupling may violate the symmetry responsible for removing the logs, these couplings may reintroduce logarithmic UV sensitivity. Therefore, it is necessary that they are small enough to not spoil the reduction in tuning. While it is not required, it is interesting to consider the region of parameter space where these couplings are so small that the decays are displaced. This could be motivated by models where the $\mathcal{Z}_{2}$ breaking is generated at the UV scale by a higher dimension operator.

The collider phenomenology of a supersymmetric vectorlike fourth generation has been studied previously (see, for example, [31,32]), and is rich. In particular, logging requires the particular slumberjack soft-mass pattern given in Eq. (3). Post discovery, detailed measurements of the mass spectrum could yield strong evidence that this mechanism is being used by nature to avoid excessive fine-tuning of the weak scale. It would be interesting to study the viability of such measurements at the HL-LHC or FCC-hh. However, since the detailed signatures are highly model dependent in a manner that is unconnected to the supersoft properties we leave the exploration of this phenomenology to future work.

Summary.-Naturalness seeds of doubt were sown when LEP measurements demonstrated precision agreement with the predictions of the standard model. They have since germinated due to the paucity of discoveries beyond the Higgs boson at the LHC and are now maturing into a naturalness crisis [33]. Our hope to experimentally access the microscopic provenance of the electroweak scale has been challenged by the interpretation of null results in the context of classic scenarios such as the MSSM and minimal composite Higgs models.

When one assumes that the electroweak hierarchy problem is tamed through the introduction of a new symmetry that commutes with the standard model gauge groups, there is a generic expectation that new colored "top-partner" states should exist in proximity to the weak scale. Since LHC null results currently imply $\tilde{m} \gtrsim 900 \mathrm{GeV}$, the resultant naïve scaling of Higgs mass corrections [Eq. (8)] might cause one to conjecture that naturalness is not a useful guide for predicting the next layer of fundamental physics. This is exacerbated if one assumes that the fundamental microscopic scales satisfy $\Lambda_{\mathrm{UV}} \gg M_{H}$ as this raises the Higgs mass parameter through large logarithmic enhancements. However, we emphasize here that this scaling is only a minimal expectation, subject to very basic underlying assumptions.

To this end we have embraced the log-enhanced contributions and, rather than attempting to banish them by reducing $\Lambda_{\mathrm{UV}}$, we have instead introduced the lumberjack fields, who screen logs through equal and opposite- sign contributions from the mediation scale $\Lambda_{\mathrm{UV}}$ down to the scale $M$ where they decouple. This significantly reduces the fine-tuning associated with the top sector-resulting in a model where a mediation scale $\Lambda_{\mathrm{UV}} \gg M_{H}$ can be just as natural as one where the mediation scale is low.

This supersoft strategy has been previously studied for the gauge sector, and is shown here to be possible for the matter sector. In the models presented here, one may have a grand unified theory-scale mediation scale and top squarks heavier than $\sim 1 \mathrm{TeV}$, consistent with current limits [34,35], with fine-tuning reduced by a factor $\mathcal{O}(30)$ as compared to the most basic MSSM expectation.

The reduced fine-tuning in this supersoft top squark model makes clear it is dangerous to over-interpret naturalness implications of null results from LHC colored particle searches, since any interpretation is highly model dependent. Nature does not have to make the minimal choice, thus it is premature to conclusively declare that high-scale SUSY is incompatible with naturalness.

This work is dedicated to the original supersoft pioneer, Ann Nelson. T. C., N. C., S. K., and M. M. are grateful to the KITP "Origin of the Vacuum Energy and Electroweak Scales" program. This research was supported in part by the National Science Foundation under Grant No. NSF PHY1748958. T. C. is supported by the U.S. Department of Energy (DOE), under Grant No. DE-SC0011640. M. M. is supported by the STFC HEP consolidated Grant No. ST/ P000681/1 and Trinity College Cambridge. N. C. and S. K. are supported by the U.S. Department of Energy (DOE) under the Early Career Award No. DE-SC0014129 and the Cottrell Scholar Program through the Research Corporation for Science Advancement. J. T. S. is supported by the STFC consolidated Grant No. ST/S505316/1.

[1] P. J. Fox, A. E. Nelson, and N. Weiner, Dirac gaugino masses and supersoft supersymmetry breaking, J. High Energy Phys. 08 (2002) 035.

[2] C. Csaki, T. Ma, and J. Shu, Maximally Symmetric Composite Higgs Models, Phys. Rev. Lett. 119, 131803 (2017).

[3] S. Blasi and F. Goertz, Softened Symmetry Breaking in Composite Higgs Models, Phys. Rev. Lett. 123, 221801 (2019).

[4] J. Scherk and J. H. Schwarz, How to get masses from extra dimensions, Nucl. Phys. B153, 61 (1979).

[5] J. Scherk and J.H. Schwarz, Spontaneous breaking of supersymmetry through dimensional reduction, Phys. Lett. 82B, 60 (1979).

[6] I. Antoniadis, S. Dimopoulos, A. Pomarol, and M. Quiros, Soft masses in theories with supersymmetry breaking by TeV compactification, Nucl. Phys. B544, 503 (1999).

[7] A. Pomarol and M. Quiros, The standard model from extra dimensions, Phys. Lett. B 438, 255 (1998).

[8] A. Delgado and M. Quiros, Supersymmetry and finite radiative electroweak breaking from an extra dimension, Nucl. Phys. B607, 99 (2001). 
[9] A. Delgado, G. von Gersdorff, and M. Quiros, Two loop Higgs mass in supersymmetric Kaluza-Klein theories, Nucl. Phys. B613, 49 (2001).

[10] R. Barbieri, G. Marandella, and M. Papucci, The Higgs mass as a function of the compactification scale, Nucl. Phys. B668, 273 (2003).

[11] R. Barbieri, L. J. Hall, G. Marandella, Y. Nomura, T. Okui, S. J. Oliver, and M. Papucci, Radiative electroweak symmetry breaking from a quasilocalized top quark, Nucl. Phys. B663, 141 (2003).

[12] S. Dimopoulos, K. Howe, and J. March-Russell, Maximally Natural Supersymmetry, Phys. Rev. Lett. 113, 111802 (2014).

[13] N. Arkani-Hamed, A. G. Cohen, and H. Georgi, Electroweak symmetry breaking from dimensional deconstruction, Phys. Lett. B 513, 232 (2001).

[14] N. Arkani-Hamed, A. G. Cohen, T. Gregoire, and J. G. Wacker, Phenomenology of electroweak symmetry breaking from theory space, J. High Energy Phys. 08 (2002) 020.

[15] Note that a very similar Lagrangian appears in [16], albeit with uncolored top sector copies and a requirement that $|M| \approx|\tilde{m}|$, which is not required for the model proposed here. Another similar action appears in the extradimensional, and hence low cutoff, model of [17], which also has a different spectrum of scalar masses.

[16] H.-C. Cheng, L. Li, E. Salvioni, and C. B. Verhaaren, Singlet scalar top partners from accidental supersymmetry, J. High Energy Phys. 05 (2018) 057.

[17] Z. Chacko, C. A. Krenke, and T. Okui, Supersymmetry in slow motion, J. High Energy Phys. 01 (2009) 050.

[18] H. Baer, V. Barger, and D. Mickelson, How conventional measures overestimate electroweak fine-tuning in supersymmetric theory, Phys. Rev. D 88, 095013 (2013).

[19] J. A. Casas, J. M. Moreno, S. Robles, K. Rolbiecki, and B. Zaldvar, What is a Natural SUSY scenario?, J. High Energy Phys. 06 (2015) 070.

[20] M. R. Buckley, A. Monteux, and D. Shih, Precision corrections to fine tuning in SUSY, J. High Energy Phys. 06 (2017) 103.

[21] L. O'Raifeartaigh, Spontaneous symmetry breaking for chiral scalar superfields, Nucl. Phys. B96, 331 (1975).

[22] N. Arkani-Hamed, M. Dine, and S. P. Martin, Dynamical supersymmetry breaking in models with a Green-Schwarz mechanism, Phys. Lett. B 431, 329 (1998).
[23] D. S. M. Alves, J. Galloway, M. McCullough, and N. Weiner, Goldstone Gauginos, Phys. Rev. Lett. 115, 161801 (2015).

[24] D. S. M. Alves, J. Galloway, M. McCullough, and N. Weiner, Models of goldstone gauginos, Phys. Rev. D 93, 075021 (2016).

[25] R. Barbieri, L. J. Hall, Y. Nomura, and V. S. Rychkov, Supersymmetry without a light Higgs boson, Phys. Rev. D 75, 035007 (2007).

[26] S. Y. Choi, M. Drees, J. Kalinowski, J. M. Kim, E. Popenda, and P. M. Zerwas, Color-octet scalars of $N=2$ supersymmetry at the LHC, Phys. Lett. B 672, 246 (2009).

[27] S. Y. Choi, J. Kalinowski, J. M. Kim, and E. Popenda, Scalar gluons and Dirac gluinos at the LHC, Acta Phys. Pol. B 40, 2913 (2009), https://www.actaphys.uj.edu.pl/fulltext? series $=$ Reg \&vol $=40 \&$ page $=2913$.

[28] K. Blum, A. Efrati, C. Frugiuele, and Y. Nir, Exotic colored scalars at the LHC, J. High Energy Phys. 02 (2017) 104.

[29] C. Alvarado, A. Delgado, and A. Martin, Constraining the $R$-symmetric chargino NLSP at the LHC, Phys. Rev. D 97, 115044 (2018).

[30] G. D. Kribs and A. Martin, Supersoft supersymmetry is super-safe, Phys. Rev. D 85, 115014 (2012).

[31] S. P. Martin, Extra vector-like matter and the lightest Higgs scalar boson mass in low-energy supersymmetry, Phys. Rev. D 81, 035004 (2010).

[32] P. W. Graham, A. Ismail, S. Rajendran, and P. Saraswat, A little solution to the little hierarchy problem: A vector-like generation, Phys. Rev. D 81, 055016 (2010).

[33] G. F. Giudice, The dawn of the post-naturalness era, in From My Vast Repertoire ...: Guido Altarelli's Legacy, edited by A. Levy, S. Forte, and G. Ridolfi (World Scientific, Singapore, 2019), pp. 267-292.

[34] M. Aaboud et al. (ATLAS Collaboration), Search for topsquark pair production in final states with one lepton, jets, and missing transverse momentum using $36 \mathrm{fb}^{-1}$ of $\sqrt{\mathrm{s}}=$ $13 \mathrm{TeV}$ pp collision data with the ATLAS detector, J. High Energy Phys. 06 (2018) 108.

[35] A. M. Sirunyan et al. (CMS Collaboration), Search for direct top squark pair production in events with one lepton, jets, and missing transverse momentum at $13 \mathrm{TeV}$ with the CMS experiment, J. High Energy Phys. 05 (2020) 032. 